DOI: 10.1007/s10357-009-1701-7

\section{Vermeidung und Sanierung von Umweltschäden}

Richtlinie 2004/35/EG

Die Republik Österreich hat dadurch gegen ihre Verpflichtungen aus der Richtlinie 2004/35/EG des Europäischen Parlaments und des Rates vom 21.4.2004 über Umwelthaftung zur Vermeidung und Sanierung von Umweltschäden verstoßen, dass sie die erforderlichen Rechts- und Verwaltungsvorschriften zur Umsetzung dieser Richtlinie nicht innerhalb der vorgeschriebenen Frist erlassen hat.

EuGH, Urteil vom 18. 6.2009-C-422/08-

[1] Mit ihrer Klage beantragt die Kommission der Europäischen Gemeinschaften, festzustellen, dass die Republik Österreich dadurch gegen ihre Verpflichtungen aus der Richtlinie 2004/35/EG des Europäischen Parlaments und des Rates vom 21.4.2004 über Umwelthaftung zur Vermeidung und Sanierung von Umweltschäden (ABl. L 143, S. 56, im Folgenden: Richtlinie) verstoßen hat, dass sie die erforderlichen Rechts- und Verwaltungsvorschriften zur Umsetzung dieser Richtlinie nicht erlassen bzw. der Kommission diese Vorschriften nicht mitgeteilt hat.

[2] Nach Art. 19 Abs. 1 Unterabs. 1 der Richtlinie setzen die Mitgliedstaaten die Rechts- und Verwaltungsvorschriften in Kraft, die erforderlich sind, um der Richtlinie spätestens bis zum 30.4.2007 nachzukommen, und setzen die Kommission unverzüglich davon in Kenntnis.

\section{Vorverfahren}

[3] Da die Kommission innerhalb der vorgeschriebenen Frist keine Nachricht betreffend die von der Republik Österreich ergriffenen Maßnahmen zur Umsetzung der Richtlinie in ihre innerstaatliche Rechtsordnung erhalten hatte, leitete sie das in Art. 226 EG vorgesehene Vertragsverletzungsverfahren ein.

[4] Mit Schreiben vom 31.5.2007 setzte die Kommission der Republik Österreich eine Frist zur Äußerung. Letztere setzte die Kommission mit Schreiben vom 31.7.2007 davon in Kenntnis, dass die Umsetzung zum einen auf Bundesebene, durch das bereits in Ausarbeitung befindliche Bundes-Umwelthaftungsgesetz, und zum anderen auf Ebene der Länder erfolgen werde, von denen einige mit der Umsetzung begonnen und Entwürfe angenommen hätten, die bis spätestens Anfang 2008 finalisiert werden sollten.

[5] Da die Kommission die Richtlinie als noch nicht umgesetzt ansah, gab sie am 31.1.2008 eine mit Gründen versehene Stellungnahme ab, in der die Republik Österreich aufgefordert wurde, die erforderlichen Maßnahmen zu ergreifen, um den Verpflichtungen aus der Richtlinie innerhalb einer Frist von zwei Monaten ab Erhalt dieser Stellungnahme nachzukommen.

[6] Mit Schreiben vom 1.4.2008 antwortete die Republik Österreich auf diese Stellungnahme und teilte der Kommission mit, dass die Umsetzung der Richtlinie sowohl auf Bundes- als auch auf Länderebene im Gange sei.

[7] Da die Kommission keine anderen Informationen erhielt, aus denen hervorging, dass alle erforderlichen Maßnahmen zur Umsetzung der Richtlinie in die österreichische Rechtsordnung endgültig ergriffen worden waren, hat sie beschlossen, die vorliegende Klage zu erheben

\section{Zur Klage}

[8] Die Republik Österreich bestreitet nicht, dass die Umsetzung der Richtlinie nicht innerhalb der vorgesehenen Frist erfolgt ist. Sie weist jedoch darauf hin, dass diese Umsetzung rechtsetzende Maßnahmen zunächst auf Bundes- und sodann auf Länderebene erfordere.

[9] Der Entwurf des Bundes-Umwelthaftungsgesetzes sei zwar schon im Mai 2007 im Ministerrat beschlossen und dem Parlament vorgelegt worden, aufgrund von Neuwahlen müsse der genannte Entwurf jedoch vom Ministerrat neu beschlossen werden. Die Gesetzesentwürfe auf Länderebene könnten also erst nach Erlass des genannten Bundesgesetzes erlassen werden.

\section{Aus den Gründen:}

[10] Dazu ist festzustellen, dass das Vorliegen einer Vertragsverletzung anhand der Lage zu beurteilen ist, in der sich der Mitgliedstaat bei Ablauf der Frist befand, die in der mit Gründen versehenen Stellungnahme gesetzt wurde, und dass später eingetretene Änderungen vom Gerichtshof nicht berücksichtigt werden können (vgl. insbesondere Urteile vom 14. 7. 2005, Kommission/Deutschland, C-433/03, Slg. 2005, I-6985, Rdnr. 32, und vom 12.3.2009, Kommission/Luxemburg, C-289/08, Slg. 2009, I-0000, Rdnr. 7).

[11] Ferner kann sich nach ständiger Rechtsprechung ein Mitgliedstaat nicht auf Bestimmungen, Übungen oder Umstände seiner internen Rechtsordnung einschließlich solcher Umstände, die sich aus seinem bundesstaatlichen Aufbau ergeben, berufen, um die Nichteinhaltung der in einer Richtlinie festgelegten Verpflichtungen und Fristen zu rechtfertigen (vgl. in diesem Sinne Urteile vom 6.7.2000, Kommission/Belgien, C-236/99, Slg. 2000, I-5657, Rdnr. 23, und vom 12.3.2009, Kommission/Belgien, C-342/08, Slg. 2009, I-0000, Rdnr. 13).

[12] Im vorliegenden Fall steht fest, dass die Republik Österreich bei Ablauf der Frist, die in der mit Gründen versehenen Stellungnahme gesetzt worden war, die erforderlichen Maßnahmen zur Umsetzung der Richtlinie in ihre innerstaatliche Rechtsordnung nicht ergriffen hatte.

[13] Demnach ist die von der Kommission erhobene Klage begründet.

[14] Daher ist festzustellen, dass die Republik Österreich dadurch gegen ihre Verpflichtungen aus der Richtlinie verstoßen hat, dass sie nicht innerhalb der vorgeschriebenen Frist die Rechts- und Verwaltungsvorschriften erlassen hat, die erforderlich sind, um der Richtlinie nachzukommen.

\section{Anforderungen des Abwägungsgebots}

\section{FStrG §17}

1. Die Anforderungen des Abwägungsgebots richten sich auch und gerade an das Berücksichtigen planerischer Alternativen.

2. Der Planfeststellungsbehörde ist bei der Alternativenprüfung ein gestuftes Verfahren gestattet, bei dem sich die Anforderungen an den Umfang der Sachverhaltsermittlung und -bewertung nach dem erreichten Planungsstand und den im Laufe des Verfahrens gewonnenen Erkenntnissen richten.

BVerwG, Beschluss vom 24. 4.2009 - 9 B $10.09-$

\section{Aus den Gründen:}

[1] Die von der Beschwerde geltend gemachte grundsätzliche Bedeutung ( $\$ 132$ Abs. 2 Nr. 1 VwGO) kommt der Rechtssache nicht zu.

[2] Die von dem Kläger zu 1 als grundsätzlich bedeutsam angesehene Frage,

„ob und inwieweit Trassenalternativen, die das Planziel, wie die gewählte Plantrasse erreichen, in jedem Planungsprozess mit der identischen Planungstiefe und dem identischen Optimierungsgrad in das Verfahren einbezogen werden müssen, um damit eine vergleichbare Entscheidungsgrundlage zu erreichen",

[3] würde sich in dem angestrebten Revisionsverfahren nicht stellen. Sie wäre nicht entscheidungserheblich, weil der Verwaltungsgerichtshof in seiner Entscheidung ausdrücklich festgestellt hat, dass der Trassenabwägungsentscheidung der Planfeststellungsbehörde eine eingehende Untersuchung und ein detaillierter Vergleich der Varianten, insbesondere auch der von dem Kläger zu 1 bevorzugten Variante Rischberg, vorausgegangen sind. Eine nochmalige verglei- 